\title{
The $n$-Port Resistive Network Synthesis from Prescribed Sensitivity Coefficients
}

\author{
M. G. GOVINDARAJULU NAIDU, K. THULASIRAMAN, MEMBER, IEEE, AND
}

M. N. S. SWAMY, SENIOR MEMBER, IEEE

\begin{abstract}
An investigation of the relationship between the sensitivity coefficients and the $K$-matrix of $n$-port networks is presented. The results available on the $K$-matrix and the adjoint network approach for sensitivity computations will form the basis of the discussions.
\end{abstract}

\section{The $P$-MATRIX}

YONSIDER a resistive $n$-port network $N$. Let $\hat{g}=$ $\left\{g_{i}\right\}$ denote the column matrix of edge conductances of $N$. We shall denote by $\hat{g}_{0}=\left\{g_{i}{ }^{0}\right\}$ the nominal value of $\hat{g}$. The sensitivity coefficient matrix $P=\left[p_{i j}\right]$ with respect to the $Y$-matrix of $N$ will be defined as follows:

$$
p_{i j}=\frac{\partial y_{i i}}{\partial g_{i}} \mid \hat{g}=\hat{g}_{0}
$$

where $y_{i i}$ is the short-circuit driving-point admittance across port $i$ of $N . p_{i j}$ will be referred to as the sensitivity coefficient of $y_{i i}$ with respect to the conductance $g_{j}$.

It may be seen that the matrix $P$ is of order $n \times e$, where $e$ is the number of edges in $N$. Without any loss of generality the graph of the network $N$ may be assumed to be complete by permitting edges with zero admittances.

It follows from the results of [1], [2] that

$$
\frac{\partial y_{i j}}{\partial g_{k}} \mid \hat{g}=\hat{g}_{0}=\left(v_{k}^{j}\right)\left(v_{k}^{i}\right)
$$

where $v_{k}^{j}\left(v_{k}{ }^{i}\right)$ is the voltage across conductance $g_{k}$ when port $j(i)$ is excited with a source of unit voltage and all the other ports are short-circuited. This is illustrated in Fig. 1. Hence we get

$$
p_{i k} \mid \hat{g}=\hat{g}_{0}=\left(v_{k}^{i}\right)^{2}
$$

as a consequence of the no amplification property of resistive networks $p_{i k} \leq 1$.

Let the port configuration $T$ of $N$ be in $p$ parts $T_{1}, T_{2}, \cdots$, $T_{p}$. Let the set of vertices in $T_{i}$ be denoted by $V_{i}$. Let $T_{0}$ be a tree of $N$ such that $T \subset T_{0}$. We shall denote by $q_{i}$ the $f$-cutset of $N$ with respect to the branch of $T$ corresponding to the port $i$. If port $i$ is in $T_{k}$, it may be seen from (3)

M. G. Govindarajulu Naidu and K. Thulasiraman are with the Department of Electrical Engineering, Indian Institute of Technology, Madras-600036, India.

Professor M. N. S. Swamy is with the Department of Electrical Engineering, Sir George Williams University, Montreal-107, Canada. Manuscript received November 14, 1972, revised November 25, 1974.

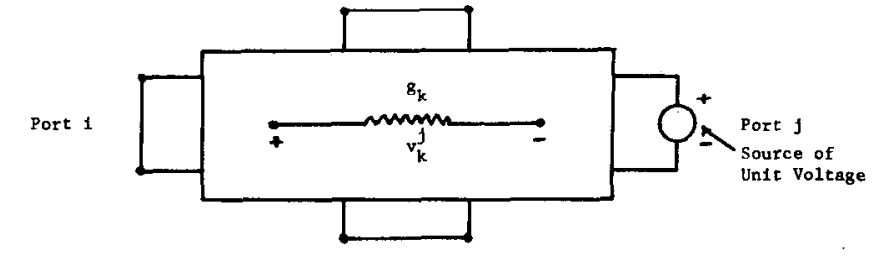

(a)

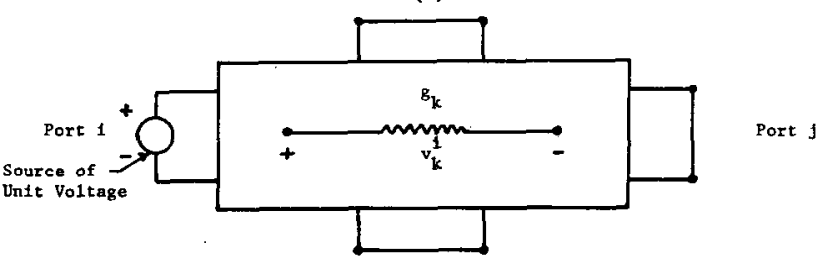

(b)

Fig. 1. (a) Resistive $n$-port network $N$ excited by unit voltage source at port $j$ with all other ports shorted. (b) Resistive $n$-port being excited by unit voltage source at port $i$ with all other ports shorted.

that for any vertices $r, s \in V_{k}$

$$
\frac{\partial y_{i i}}{\partial g_{r s}} \mid \hat{g}=\hat{g}_{0}= \begin{cases}1, & \text { if } e_{r s} \in q_{i} \\ 0, & \text { if } e_{r s} \notin q_{i}\end{cases}
$$

where $e_{r s}$ is the edge connecting vertices $r$ and $s$, and $g_{r s}$, is the conductance of $e_{r s}$.

In view of (4), the matrix $P$ can be partitioned as follows:

$$
P=\left[P_{A} \vdots P_{B}\right]
$$

where

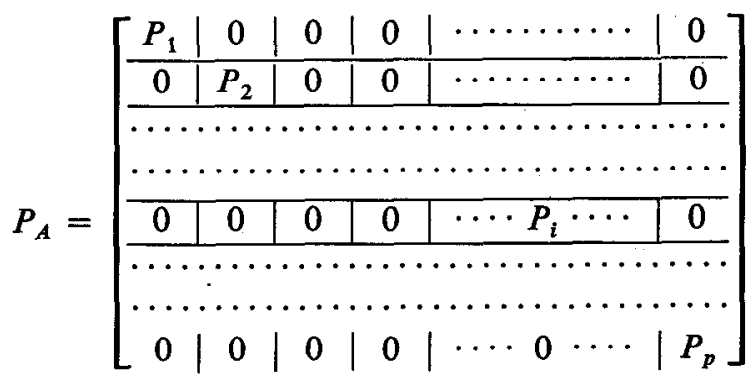

with a) the rows of $P_{i}(i=1,2, \cdots, p)$ corresponding to the ports in $T_{i}$ and the columns corresponding to the conductances $g_{r s}$, where the vertices $r, s \in V_{i}$, and b) the columns of $P_{B}$ corresponding to the conductances $g_{r s}$ where $r \in V_{k}$ and $s \in V_{j}, k \neq j$.

It may be seen that $P_{i}$ is the $f$-cutset matrix with respect to $T_{i}$ of the complete graph on the vertices of $V_{i}$. Hence given any $P$-matrix, each $T_{i}$ and hence the port configuration of $N$ can be determined. 


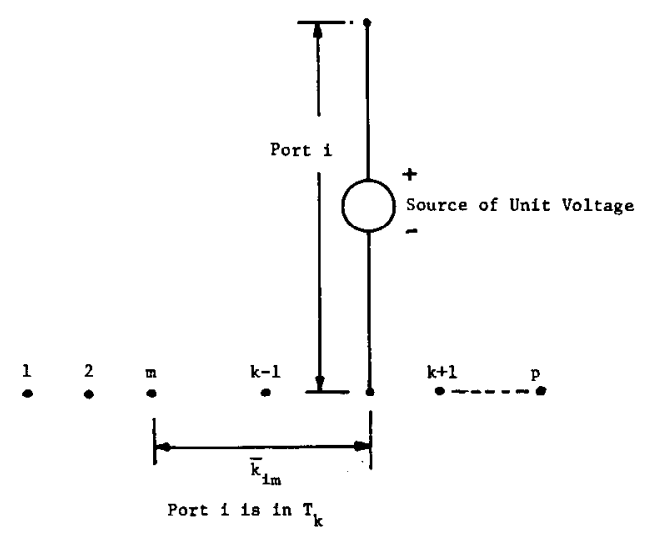

Fig. 2. The $(p+1)$ distinct vertices of network $N$ when port $i \in T_{k}$ is excited by unit voltage source with all other ports shorted.

\section{ReLATION BetWeEN $P$ - AND $K$-MATRICES}

It may be recalled [3], [4] that the $(i, j)$ entry $k_{i j}$ of the $K$-matrix, called the potential factor of port $j$ with respect to port $i$, is defined as equal to the potential of the positive reference terminal of port $j$ with respect to the negative reference terminal of port $i$, when port $i$ is excited with a source of unit voltage and all the other ports are shortcircuited. Further if port $i$ is in $T_{k}$ and is excited with unit voltage and all the other ports are short-circuited, then the potentials of all the ports in any $T_{m}, m \neq k$, with respect to the negative reference terminal of port $i$ will be equal. Hence the potential factors of all the ports in $T_{m}$, with respect to port $i$ will be equal. Let the common value of these potential factors be denoted by $\bar{K}_{i m}$. It can be easily shown [4] that after rearranging its rows and columns, the $K$-matrix can be partitioned as

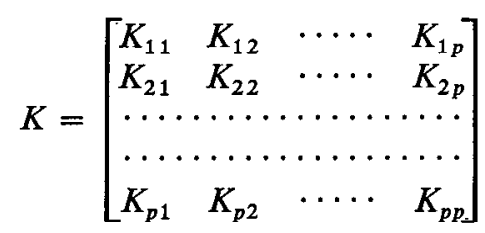

where

a) the rows and columns of $K_{i j}$ correspond to the ports in $T_{i}$ and $T_{j}$, respectively;

b) each submatrix $K_{i i}$ consists of 1's and 0's only and is uniquely specified by the configuration of $T_{i}$;

c) all the entries in any row of $K_{q m}$ will be equal, and

d) the potential factors $\bar{K}_{i m}$, for all $i \in T_{q}$, completely specify $K_{q m}$.

\section{Theorem}

Given the $P$-matrix of an $n$-port network $N$, the port configuration $T$ and the $K$-matrix of $N$ can be uniquely determined.

\section{Proof}

The submatrix $P_{i}$ of the given $P$-matrix, as mentioned earlier, completely specifies the configuration of $T_{i}$ and hence the submatrix $K_{i i}$ of the required $K$-matrix. Thus given $P$, the $K_{i i}$ submatrices of the $K$-matrix can be obtained.

Let port $i \in T_{k}$, of the $n$-port network $N$ be excited with unit voltage and all the other ports be short-circuited. Then there will be $(p+1)$ distinct vertices in the network $N$ as shown in Fig. 2. The set of ports in $T_{m}$ is represented in Fig. 2 by the vertex $m$. The potential of this vertex with respect to the negative reference terminal of port $i$ is equal to $\bar{K}_{i m}$. Consider the entry in the $i$ th row of $P$ corresponding to any conductance connecting the negative reference terminal of port $i$ and a vertex in the set $V_{m}$. Since by (3) this entry is equal to $\left(\bar{K}_{i m}\right)^{2}$, we can easily evaluate $\bar{K}_{i m}$. Thus all the potential factors $\bar{K}_{i m}, m=1,2, \cdots, p, m \neq k$, may be evaluated using the entries in row $i$ of $P$. Repeating this for all the ports in $T_{k}$, the submatrix $K_{k m}$ of the required $K$-matrix can be determined.

Thus we see from the given matrix all the $K_{i i}$ and $K_{i j}$, $j \neq i$ submatrices of the $K$-matrix can be obtained and hence the theorem.

Since the procedures for realizing (whenever possible) a resistive $n$-port network having a prescribed $K$-matrix is already available, realization of a $P$-matrix may be considered complete with the determination of the relevant $K$-matrix from the given $P$-matrix. The procedures given in [5] for the special case of $(n+2)$-node networks or the general procedures given in [4] may then be followed to realize the $K$-matrix. If the $K$-matrix derived from a given $P$-matrix is not realizable, then the $P$-matrix itself is not realizable. Simple necessary and sufficient conditions to test the realizability of a $P$-matrix are not given here, since such conditions are not available in the case of a $K$-matrix too.

Though the discussions in [4] and [5] are restricted to $n$-port networks having no internal vertices, the procedures given in these papers can be easily extended to networks with internal vertices by defining potential factors for all internal vertices too as is done in [6].

\section{EXAMPLE}

Let it be required to realize the matrix given below as the $P$-matrix of a 4-port network constructed on the resistive 


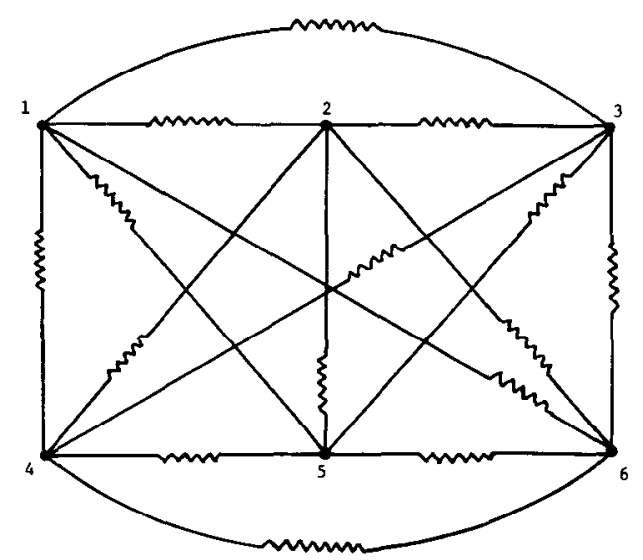

Fig. 3. Resistive network for the example.

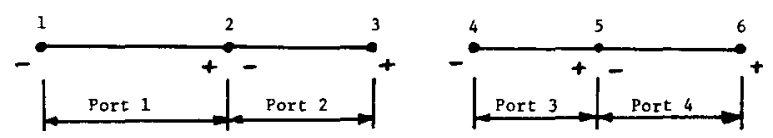

Fig. 4. Port configuration of 4-port network constructed on resistive network of Fig. 3.

network shown in Fig. 3:

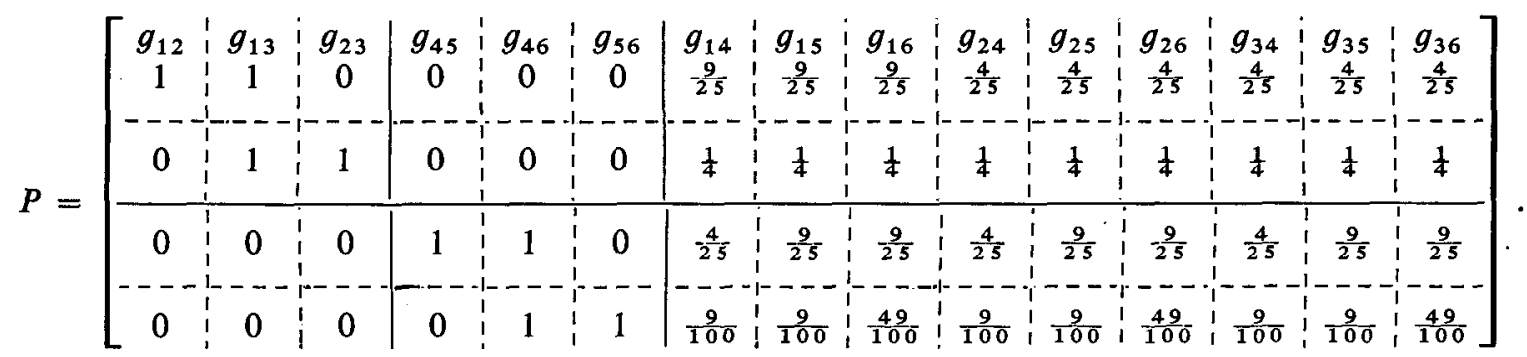

Partitioning the matrix $P$ as indicated, it may be shown easily that the port configuration of the 4-port network will be as in Fig. 4. Let $V_{1}=\{1,2,3\}$, and $V_{2}=\{4,5,6\}$. The entry in the first row and the column corresponding to the conductance $g_{14}$ is equal to $\left(\bar{K}_{12}\right)^{2}$. Hence we get $\bar{K}_{12}=$ $k_{13}=k_{14}=0.60$. Similarly, $\bar{K}_{22}, \bar{K}_{31}$, and $\bar{K}_{41}$ can be obtained from the relevant entries in the columns corresponding to the conductances $g_{24}, g_{14}, g_{15}$, respectively. Thus we get $\bar{K}_{22}=k_{23}=k_{24}=0.50 ; \quad \bar{K}_{31}=k_{31}=$ $k_{32}=0.40$; and $\bar{K}_{41}=k_{41}=k_{42}=0.30$. The $K$-matrix of the required 4-port network is then equal to

$$
K=\left[\begin{array}{c:c|c:c}
1.0 & 1.0 & 0.60 & 0.60 \\
0 & 1.0 & 0.50 & 0.50 \\
\hline 0.40 & 0.40 & 1.0 & 1.0 \\
0.30 & 0.30 & 0 & 1.0
\end{array}\right] .
$$

Using the procedure given in [4], the conductances $\left(g_{i j}\right)_{p}$ of a pading 4-port network $N_{p}$ having the foregoing $K$-matrix can be obtained as

$$
\begin{gathered}
G_{p}=\operatorname{diag}\left\{g_{12} g_{13} g_{14} g_{15} g_{16} g_{23} g_{24} g_{25} g_{26} g_{34}\right. \\
\left.g_{35} g_{36} g_{45} g_{46} g_{56}\right\}_{p} \\
=\operatorname{diag}\{-8-4048824-101226601030 \\
-12-36-6\}_{p} .
\end{gathered}
$$

The conductances of a suitable network of departure $N_{d}$ can then be obtained (using (11) and (12) of [5]) as

$$
\begin{aligned}
& G_{d}=\operatorname{diag}\left\{g_{12} g_{13} g_{14} g_{15} g_{16} g_{23} g_{24} g_{25} g_{26} g_{34}\right. \\
& \left.g_{35} g_{36} g_{45} g_{46} g_{56}\right\}_{d}
\end{aligned}
$$

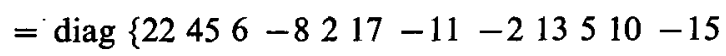

$204015\}_{d}$.

The parallel combination $N$ of $N_{p}$ and $N_{d}$ will contain no negative conductances and will realize the matrix $K$ and hence the matrix $P$. The conductances of $N$ are given by

$$
\begin{array}{r}
G=\operatorname{diag}\left\{g_{12} g_{13} g_{14} g_{15} g_{16} g_{23} g_{24} g_{25} g_{26} g_{34} g_{35}\right. \\
\left.g_{36} g_{45} g_{46} g_{56}\right\}_{N}
\end{array}
$$

$$
=\operatorname{diag}\{1455402671019652015849\}_{N} \text {. }
$$

\section{ConCLUSION}

In conclusion, it may be pointed out that the adjoint network approach for computation of sensitivities has helped in discovering the interesting relationship that exists between the sensitivity coefficients and the $K$-matrix. This relationship was not obvious when the $K$-matrix was first introduced. 
We now indicate the applicability of the results of this paper to the problem of design of minimum sensitivity resistive $n$-port networks.

Given the matrix $Y$, each edge conductance $g_{i j}$ of a network realizing $Y$ can be expressed as [4]

$$
g_{i j}=f_{i j}\left(K_{1}, K_{2}, \cdots, K_{1} ; S_{1}, S_{2}, \cdots, S_{m}\right)
$$

where $K_{i}$ 's are elements of the $K$-matrix and $S_{i}$ 's are quantities related to the network required.

A significant feature of (5) is that $f_{i j}$ is a linear combination of $S_{i}$ 's for a given choice of $K_{i}^{\prime}$ s.

The problem of minimum sensitivity resistive $n$-port network design is to minimize

$$
\phi=\sum_{r, s} \sum_{i, j}\left|\frac{g_{i j}}{y_{r s}} \cdot \frac{\partial y_{r s}}{\partial g_{i j}}\right|^{2}
$$

subject to

$$
\begin{array}{rlrl}
g_{i j} & \geq 0, & & \text { for all } i, j \\
S_{i} & \geq 0, & & \text { for all } i=1,2, \cdots, m \\
0<K_{i}<1, & & \text { for all } i=1,2, \cdots, 1 .
\end{array}
$$

Each term in $\phi$ is the square of the magnitude of the normalized sensitivity of transfer function $y_{r s}$ with respect to conductance $g_{i j}$. Note that normalized sensitivity is different from the sensitivity coefficient defined in the paper.

It can be seen from the results of the paper that $\partial y_{r s} / \partial g_{i j}$ is a function of $K_{i}$ 's only. Hence $\phi$ is a function of $K_{i}$ 's and $S_{i}$ 's. The aforementioned problem can be solved using an existing nonlinear programming technique [7].

We wish to point out that for a given choice of $K_{i}$ 's, $\phi$ is quadratic in $S_{i}$ 's, since each $g_{i j}$ is then a linear combination of $S_{i}$ 's. One may be able to take advantage of this fact in finding $K_{i}$ 's and $S$ 's which minimize $\phi$.

\section{REFERENCES}

[1] S. W. Director and R. A. Rohrer, "Automated network designThe frequency domain case," IEEE Trans. Circuit Theory, vol. CT-16, pp. 330-337, Aug. 1969.

[2] S. W. Director and R. A. Rohrer, "On the design of resistance n-port networks by digital computer," IEEE Trans. Circuit Theory, vol. CT-16, pp. 337-346, Aug. 1969 .

[3] A. Lempel and I. Cederbaum, " $K$-matrix of $n$-port networksAnalysis and synthesis," IEEE Trans. Circuit Theory, vol. CT-16, Nov. 1969 , pp. $471-483$.

[4] P. Subbarami Reddy and K. Thulasiraman, "Analysis and synthesis of the $K$ - and $Y$-matrices of resistive $n$-port networks," presented at the Asilomar Conf. on Circuits and Systems, Monterey, Calif., Nov. 1971

[5] P. Subbarami Reddy, V. G. K. Murti, and K. Thulasiraman, "Realisation of modified cut-set matrix and applications," IEEE Trans. Circuit Theory, vol. CT-17, pp. 475-486, Nov. 1970.

[6] M. N. S. Swamy and K. Thulasiraman, "Realisation of the $A$ matrix of R.L.C. networks," presented at the 1971 IEEE Int. Symp. on Circuit Theory, London, England, Sept. 1971.

[7] G. Hadley, Nonlinear and Dymanic Programming. London, England: Addison-Wesley.

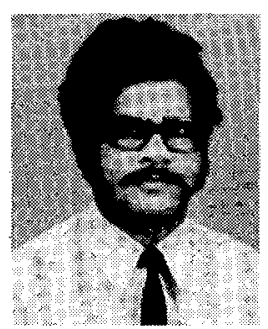

M. G. Govindarajulu Naidu was born in Aragonda (Chittoor District), Andhra Pradesh, India, on July 1, 1938. He received the B.E. degree in electrical engineering from $\mathrm{Sr}$ Venkateswara University in 1960 and the M.E. degree (Honors) in control systems engineering from the University of Roorkee, Roorkee, India, in 1967.

He worked as Junior Electrical Engineer, from 1960 to 1963 on the Andra Pradesh State Electricity Board. He was a Lecturer in the Electrical Engineering Department at Government Engineering College, Anantapur, from 1963 to 1964, and a Technical Teacher's Trainee (sponsored by the Indian government) at the University of Roorkee, from 1964 to 1967 . He served as Head of the Electrical Engineering Section in the Department of Technical Education for the government of Andhra Pradesh, from 1967 to 1971. Since 1971, he has been working for the Ph.D. degree in electrical engineering at the Indian Institute of Technology, Madras. His current research interests are networks and systems theory and graph theory.

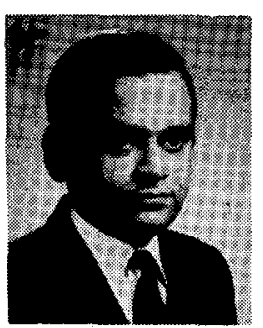

K. Thulasiraman (M'72) was born in Ammayappan, Tamil Nadu, India, on June 9, 1942. He received the B.E. and M.Sc. degrees in electrical engineering from the University of Madras, Madras, India, in 1963 and 1965, respectively, and the $\mathrm{Ph} . \mathrm{D}$. degree in electrical engineering from the Indian Institute of Technology (I.I.T.), Madras, in 1968.

In September, 1965, he joined the Department of Electrical Engineering at the I.I.T. From September 1970 to May 1972, he was a Post-doctoral Fellow at Sir George Williams University, Montreal, Canada. Since June 1973, he has been with the Computer Center of the I.I.T. where he is presently an Associate Professor. His current research interests are in the area of networks and systems theory, graph theory, and combinatorial mathematics.

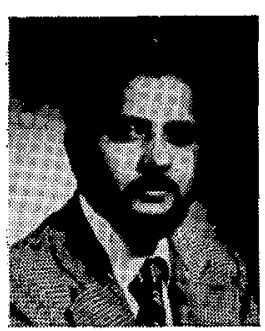

M. N. S. Swamy (S'59-M'62-SM'74) was born on April 7, 1935. He received the B.Sc. (Honors) degree in mathematics from Mysore, India, in 1954, the Diploma in electrical communication engineering from the Indian Institute of Science, Bangalore, in 1957, and the M.Sc. and Ph.D. degrees in electrical engineering from the University of Saskatchewan, Saskatoon, Saskatchewan, Canada, in 1960 and 1963, respectively.

He worked as a Senior Research Assistant at the Indian Institute of Science until 1959, when he began graduate study at the University of Saskatchewan. In 1963, he returned to India to work at the Indian Institute of Technology, Madras. From 1964 to 1965, he was an Assistant Professor of Mathematics at the University of Saskatchewan. He has also taught as Professor of Electrical Engineering at Nova Scotia Technical College, Halifax, and the University of Calgary, Calgary, Alberta, Canada. He is now Chairman of the Department of Electrical Engineering, Concordia University, Montreal, Canada. He has published a number of papers on number theory, semiconductor circuits, control systems, and network theory. He is also an Associate Editor of the Fibonacci Quarterly. 